Louisiana State University

LSU Digital Commons

$1-1-2017$

\title{
Reanalysis of LIGO black-hole coalescences with alternative prior assumptions
}

\author{
Davide Gerosa \\ California Institute of Technology \\ Salvatore Vitale \\ LIGO, Massachusetts Institute of Technology \\ Carl Johan Haster \\ L'Institut Canadien d'Astrophysique Théorique \\ Katerina Chatziioannou \\ L'Institut Canadien d'Astrophysique Théorique \\ Aaron Zimmerman \\ L'Institut Canadien d'Astrophysique Théorique
}

See next page for additional authors

Follow this and additional works at: https://digitalcommons.Isu.edu/physics_astronomy_pubs

\section{Recommended Citation}

Gerosa, D., Vitale, S., Haster, C., Chatziioannou, K., Zimmerman, A., González, G., \& Hynes, R. (2017). Reanalysis of LIGO black-hole coalescences with alternative prior assumptions. Proceedings of the International Astronomical Union, 13 (S338), 22-28. https://doi.org/10.1017/S1743921318003587 


\section{Authors}

Davide Gerosa, Salvatore Vitale, Carl Johan Haster, Katerina Chatziioannou, Aaron Zimmerman, G. González, and R. Hynes 


\section{UNIVERSITYOF BIRMINGHAM \\ University of Birmingham Research at Birmingham}

\section{Reanalysis of LIGO black-hole coalescences with alternative prior assumptions}

Gerosa, Davide; Vitale, Salvatore; Haster, Carl-Johan; Chatziioannou, Katerina; Zimmerman, Aaron

DOI:

10.1017/S1743921318003587

License:

None: All rights reserved

\section{Document Version}

Peer reviewed version

Citation for published version (Harvard):

Gerosa, D, Vitale, S, Haster, C-J, Chatziioannou, K \& Zimmerman, A 2017, 'Reanalysis of LIGo black-hole coalescences with alternative prior assumptions', Proceedings of the International Astronomical Union, vol. 13, no. Symposium 338, pp. 22-28. https://doi.org/10.1017/S1743921318003587

Link to publication on Research at Birmingham portal

\section{Publisher Rights Statement:}

Checked for eligibility: 17/05/2019

This document is the accepted manuscript version of a published work that appeared in final form in Proceedings of the International Astronomical Union. ( International Astronomical Union. The final version of record can be found at: https://doi.org/10.1017/S1743921318003587

\section{General rights}

Unless a licence is specified above, all rights (including copyright and moral rights) in this document are retained by the authors and/or the copyright holders. The express permission of the copyright holder must be obtained for any use of this material other than for purposes permitted by law.

- Users may freely distribute the URL that is used to identify this publication.

- Users may download and/or print one copy of the publication from the University of Birmingham research portal for the purpose of private study or non-commercial research.

- User may use extracts from the document in line with the concept of 'fair dealing' under the Copyright, Designs and Patents Act 1988 (?)

- Users may not further distribute the material nor use it for the purposes of commercial gain.

Where a licence is displayed above, please note the terms and conditions of the licence govern your use of this document.

When citing, please reference the published version.

Take down policy

While the University of Birmingham exercises care and attention in making items available there are rare occasions when an item has been uploaded in error or has been deemed to be commercially or otherwise sensitive.

If you believe that this is the case for this document, please contact UBIRA@lists.bham.ac.uk providing details and we will remove access to the work immediately and investigate. 


\title{
Reanalysis of LIGO black-hole coalescences with alternative prior assumptions
}

\author{
Davide Gerosa $^{1} \dagger$, Salvatore Vitale ${ }^{2}$, Carl-Johan Haster ${ }^{3}$, \\ Katerina Chatziioannou ${ }^{3}$ and Aaron Zimmerman ${ }^{3}$ \\ ${ }^{1}$ TAPIR 350-17, California Institute of Technology, \\ 1200 E California Boulevard, Pasadena, CA 91125, USA \\ ${ }^{2}$ LIGO, Massachusetts Institute of Technology, \\ 77 Massachusetts Avenue, Cambridge, MA 02139, USA \\ ${ }^{3}$ Canadian Institute for Theoretical Astrophysics, University of Toronto, \\ 60 St. George Street, Toronto, ON M5S 3H8, Canada \\ dgerosa@caltech.edu
}

\begin{abstract}
We present a critical reanalysis of the black-hole binary coalescences detected during LIGO's first observing run under different Bayesian prior assumptions. We summarize the main findings of Vitale et al. (2017) and show additional marginalized posterior distributions for some of the binaries' intrinsic parameters.
\end{abstract}

These findings were presented at IAU Symposium 338, held on October 16-19, 2017 in Baton Rouge, LA, USA.

\section{The incredible story of the first LIGO and Virgo events}

The first two observing runs of the advanced gravitational wave (GW) detectors LIGO and Virgo (Aasi et al. 2015; Acernese et al. 2015) are an incredible story of scientific achievement (and possibly a pinch of luck).

The first observing run (O1) lasted from September 2015 to January 2016, during which the two LIGO detectors in Hanford, WA and Livingston, LA collected about $\sim 51.5$ days hours of coincident data. Three detections were made during this first data taking period, all from black hole (BH) binary systems (Abbott et al. 2016a). A few days before the official start of the scientific run, the strong event GW150914 from a BH binary with total mass $\sim 65 M_{\odot}$ hit the two LIGO detectors with a signal-to-noise ratio (SNR) of $\sim 24$ (Abbott et al. 2016c). This first landmark observation of GWs is one of the greatest achievements in modern science and crowned with success more than 50 years of experimental and theoretical effort in building and operating GW detectors. A few months later, on a Christmas day evening in the US, LIGO detected a second signal from a lighter BH binary of $\sim 24 M_{\odot}$, GW151226 (Abbott et al. 2016b). A weaker and less significant event, LVT151012, was also detected during O1. The probability that LVT151012 was not instrumental noise is $87 \%$ (Abbott et al. 2016a). It turns out this is not enough to qualify for the "GW" stamp, and the event was designated to be a LIGO/Virgo trigger.

The second observing run (O2) lasted from November 2016 to August 2017. The earliest two detections announced during O2 curiously mirrored the O1 results. First, GW17014 was detected from a BH binary of $\sim 50 M_{\odot}$ similar to that of GW150914 (Abbott et al. 2017a). Later on, GW170608 was detected from a BH binary of $\sim 19 M_{\odot}$ which resembles GW151226 (Abbott et al. 2017b). The real surprises came towards the end of the data

$\dagger$ Einstein Fellow 
taking period. GW170814 was the first event detected simultaneously by the two LIGO detectors in the US and the Virgo interferometer in Europe (Abbott et al. 2017c). Adding the third detector to the network allowed for a drastic reduction of the sky location error box (from $\sim 1000 \mathrm{deg}^{2}$ to $\sim 100 \mathrm{deg}^{2}$ ), opening the possibility of rapid electromagnetic follow-up campaigns. In a remarkable twist of events, this possibility was realized only three days later, when GW170817 hit the LIGO detectors and was not confidently detected by Virgo (Abbott et al. 2017d). Virgo however, was taking data at the time, meaning the signal came from close to the blind spot of the interferometer. This allowed for an incredibly accurate sky localization within $\sim 27 \mathrm{deg}^{2}$. The estimated masses of GW170817 are compatible with those of neutron stars, whose merger is expected to produce a variety of electromagnetic signatures. Indeed, the same event was seen in gamma rays just $\sim 1.7 \mathrm{~s}$ after merger and, in just a few hours, several observatories identified an optical transient in NGC 4993, a lenticular galaxy at a mere distance of $\sim 40 \mathrm{Mpc}(\mathrm{Ab}-$ bott et al. 2017e). This triggered an extensive follow-up campaign in all electromagnetic bands, providing us unique insights on neutron star mergers, short gamma-ray bursts, and kilonovae.

The parameters of all LIGO/Virgo events were estimated using powerful statistical pipelines which inevitably include prior assumptions. Here we present a critical reanalysis of the three O1 detections (GW150914, GW151226, and LVIT151012) under different Bayesian priors, summarizing results presented by Vitale et al. (2017). We repeat some references and context from Vitale et al. (2017) but refer the reader to that work for full details. This was the first independent reanalysis of the public LIGO data that made astrophysical inferences about the sources of the signals. This study pioneered the use of the scientific products released by the LIGO and Virgo Collaboration (losc.ligo.org, Vallisneri et al. 2015) to uncover finer details of these landmark discoveries.

\section{Bayesian statistics: a magnifying lens for experimental data}

Current BH binary data were analyzed using Bayesian statistics. In a nutshell, Bayesian statistics aims at improving our understanding of any given phenomenon (like BH mergers) by updating previous knowledge in light of new data. This principle is encoded in Bayes' theorem

$$
P(\theta \mid d)=\frac{P(d \mid \theta) p(\theta)}{\int P(d \mid \theta) p(\theta) d \theta} .
$$

The posterior probability $P(\theta \mid d)$ of measuring parameters $\theta$ in light of some new data $d$ is proportional to both the likelihood $P(d \mid \theta)$ of measuring $d$ and our prior beliefs $p(\theta)$. Much like a magnifying lens allows to spot finer details, Bayesian statistics is a natural procedure to make the information brought by the data evident against the background of our prior knowledge. Crucially, such background needs to be specified.

In GW research, we all carry a monumental prior, namely the theory of General Relativity (GR). GR, indeed, has passed all current experimental tests with flying colors. It is therefore reasonable to assume that GR is an accurate description of reality, even if it may not be the ultimate theory of gravity. This does not mean that GR is not put to the test with GW data, but rather that it is more reasonable to attempt measurements of deviations from GR, rather than measuring the absolute theory of gravity. This approach just reflects previous experience, corroborated by $\sim 100$ years of data, that saw GR coming out of any experimental test stronger than before. 


\section{What prior knowledge could go into a black hole analysis?}

Astrophysical BHs (in GR at least) are fully characterized by two quantities, namely their mass and spin. A BH binary is therefore described by eight intrinsic parameters $\theta=\left\{m_{1}, m_{2}, \boldsymbol{\chi}_{\mathbf{1}}, \boldsymbol{\chi}_{\mathbf{2}}\right\}$. Let us describe the direction of each spin vector $\boldsymbol{\chi}_{\boldsymbol{i}}$ with a polar angle $\theta_{i}$ measured from the binary's orbital angular momentum $\mathbf{L}$ and an azimuthal angle $\phi_{i}$ measured in the orbital plane. The relative orientations of the spins and the orbital angular momentum depend on the three angles $\theta_{1}, \theta_{2}$ and $\Delta \Phi=\phi_{2}-\phi_{1}$.

Prior distributions have to be specified on all these parameters when analyzing $\mathrm{BH}$ coalescence data. All LIGO/Virgo analysis were performed with uniform prior distributions in $\left(m_{1}, m_{2}\right), \chi_{1}, \chi_{2}, \cos \theta_{1}, \cos \theta_{2}, \phi_{1}$ and $\phi_{2}$ at the reference frequency of $f_{\text {ref }}=20$ $\mathrm{Hz}$ (Abbott et al. 2016a,b,c, 2017a,b,c,d). This is a very reasonable approach: we have never observed BHs in binaries before, so there is no reason to prefer a particular mass or spin range. However, this is surely not the only reasonable approach. For instance, spins are vectors, not scalar. One might prefer to assume spin vectors uniform in volume, rather than uniform in magnitude and isotropic in direction. We also have solid understanding that massive stars end their lives as BHs, following core collapse. Should we insert this piece of information into our BH analysis? The distribution of stellar masses follow a power-law distribution $p(m) \propto m^{\alpha}$ with $\alpha \sim-2.3$. Could this be a good candidate for a $\mathrm{BH}$ mass prior?

Vitale et al. (2017) first tried to address some of these issues by fully reanalyzing the O1 LIGO data with different prior assumptions. Their prior choices are summarized as follows:

$\left(P_{1}\right)$ Masses are uniform, spin magnitudes are uniform, spin directions are isotropic. This is the standard prior choice made by the LIGO/Virgo Collaboration.

$\left(P_{2}\right)$ Masses are uniform, spin magnitudes are uniform in rotational energy, spin directions are isotropic.

$\left(P_{3}\right)$ Masses are uniform, spin magnitudes are uniform in volume, spin directions are isotropic.

$\left(P_{4}\right)$ Masses are uniform, spin magnitudes are drawn from a bimodal distribution peaked at low and large spins, spin directions are isotropic.

$\left(P_{5}\right)$ Masses are uniform, spin magnitudes are uniform, spin directions are preferentially aligned with $\mathbf{L}$ (i.e. small $\theta_{i}$ ).

$\left(P_{6}\right)$ Masses of the primary BHs are drawn from the stellar initial-mass function, secondary masses are uniform, spin magnitudes are uniform and spin directions are isotropic;

$\left(P_{7}\right)$ Masses of the primary BHs are drawn from the stellar initial-mass function, mass ratio is drawn from a logistic distribution, spin magnitudes are uniform and spin directions are isotropic.

$\left(P_{8}\right)$ Masses are uniform, spins magnitudes are preferentially low, spins directions are isotropic.

As detailed in Vitale et al. (2017), these choices leverage some know astrophysical properties of stellar-mass BHs and/or theoretical predictions on their population in binaries.

\section{Marginalized posteriors under different priors}

In Figs. 1 and 2 we shows marginalized posterior distributions for a variety of intrinsic parameters that characterize the observed $\mathrm{BH}$ events and all our prior choices $P_{1}-P_{8}$. Details on the data analysis technique are reported by Vitale et al. (2017). The two component masses $m_{1}$ and $m_{2}$ can be combined into total mass $M=m_{1}+m_{2}$, mass 

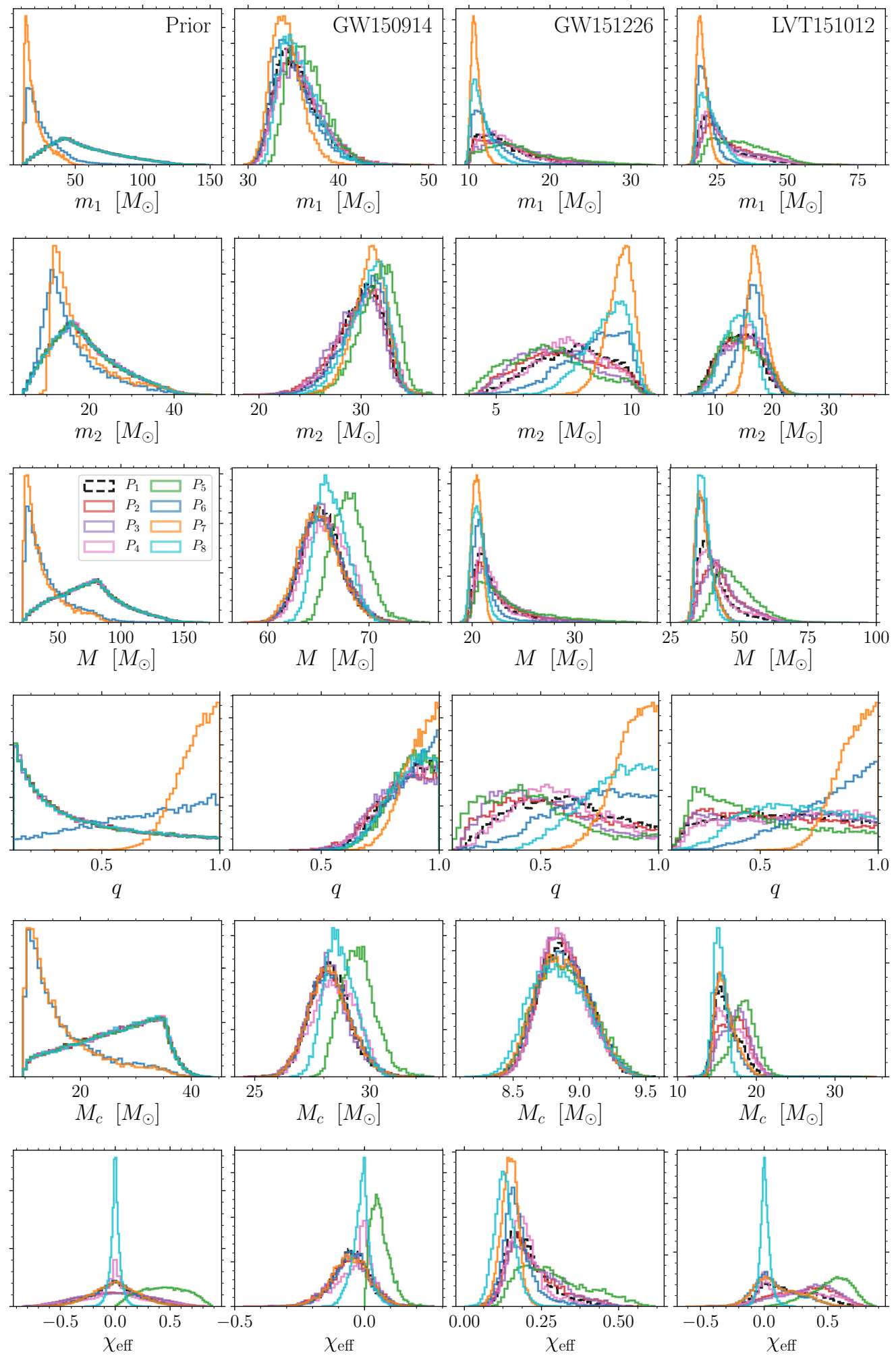

Figure 1. Prior (left panels) and posterior densities for GW150914, GW151226 and LVT151012 (second, third and fourth column from the left) under a variety of prior assumptions $P_{1}-P_{8}$. We show marginalized distributions for the BH component masses $m_{1}$ and $m_{2}$, total mass $M$, mass ratio $q$, chirp mass $M_{c}$ and effective spin $\chi_{\text {eff }}$. The $\chi_{\text {eff }}$ panels were already presented by Vitale et al. (2017). 

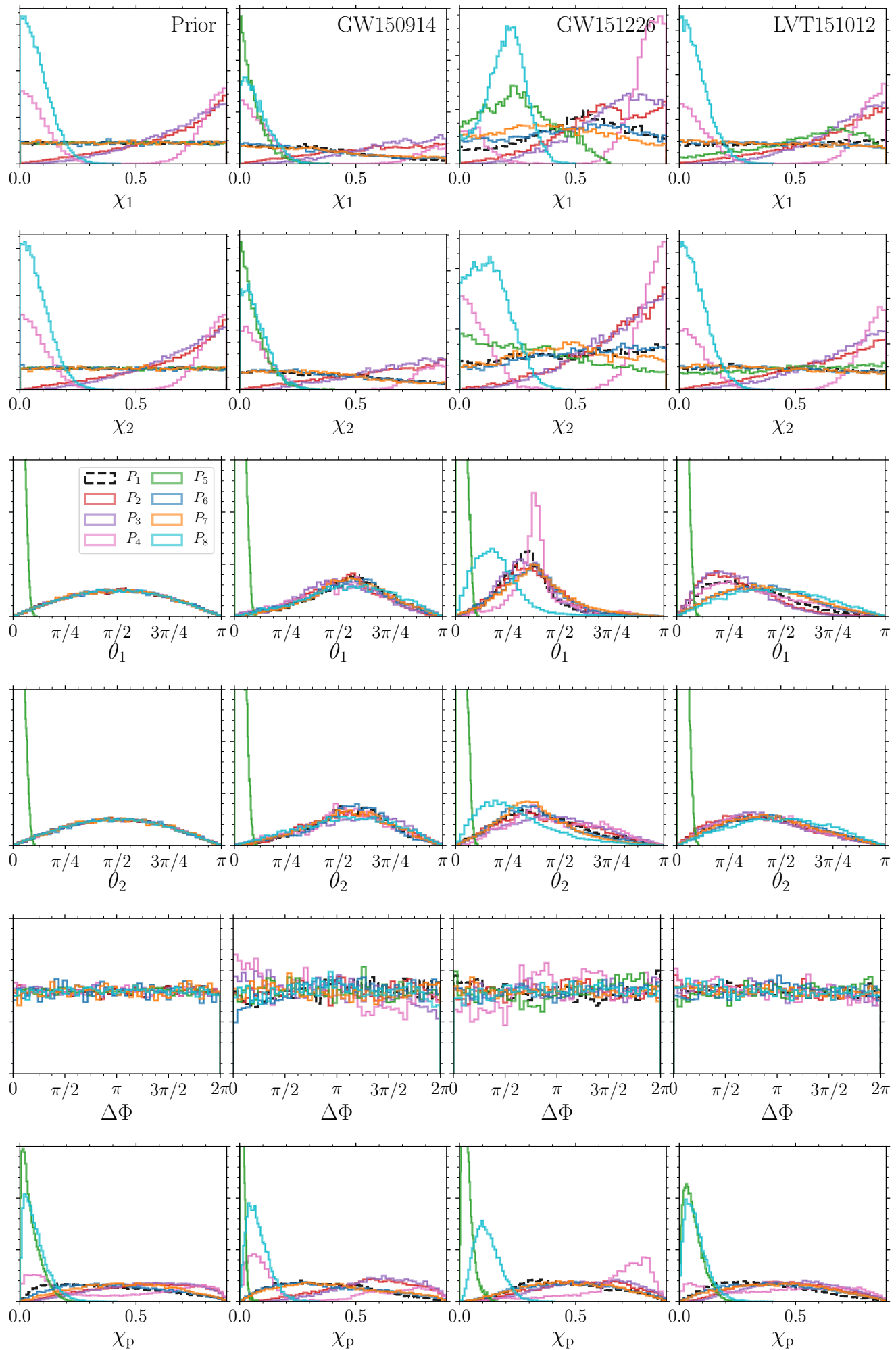

Figure 2. Prior (left panels) and posterior densities for GW150914, GW151226 and LVT151012 (second, third and fourth column from the left) under a variety of prior assumptions $P_{1}-P_{8}$. We show marginalized distributions for the $\mathrm{BH}$ spin magnitudes $\chi_{1}$ and $\chi_{2}$, the tilt angles $\theta_{1}$ and $\theta_{2}$ measured from the orbital angular momentum, the angle between the projections of the two spins onto the orbital plane $\Delta \Phi=\phi_{2}-\phi_{1}$, and the precession parameter $\chi_{p}$. Spin angles are reported at the reference frequency $f_{\text {ref }}=20 \mathrm{~Hz}$. 
ratio $q=m_{2} / m_{1} \leqslant 1$ and chirp mass $M_{c}=\left(m_{1} m_{2}\right)^{3 / 5} / M^{1 / 5}$. The best measured spin quantity is the effective spin $\chi_{\text {eff }}=\left(m_{1} \chi_{1} \cos \theta_{1}+m_{2} \chi_{2} \cos \theta_{2}\right) / M$; spin precession is implemented into the waveform template used in this analysis through a single parameter $\chi_{p}$.

Some of the inferred physical properties are robust under the choice of the Bayesian priors. For instance:

(a) non-spinning BHs for GW151226 are excluded at > 90\% credible interval;

(b) conversely, GW150914 and LVT151012 can be described by $\chi_{1}=\chi_{2}=0$;

(c) mass ratios $q \lesssim 0.5$ for GW150914 are excluded at $>90 \%$ credible interval;

(d) the $90 \%$ credible intervals in $M_{c}$ change by $<1 M_{\odot}$ in all cases;

On the other hand, other parameters strongly depend on the prior. Notably, this includes component masses $m_{i}$ and spin magnitudes $\chi_{i}$. The spin angles $\theta_{1}, \theta_{2}, \Delta \Phi$ and the spin precession parameter $\chi_{p}$ cannot be constrained meaningfully, under any of the priors tested here. We note, however, that this could be due to the waveform approximant used by Vitale et al. (2017) which only implements precession through a single effective spin. In general, prior effects are more severe for low SNR events like LVT151012, where data are less informative. A thorough discussion of these results (including odds ratio calculations) has been presented by Vitale et al. (2017). We encourage the reader to compare the findings spelled out by Vitale et al. (2017) with Fig. 1 and 2 of these proceedings.

\section{Was it necessary?}

Once a posterior $P(\theta \mid d)$ has been obtained from a reference prior $p(\theta)$ (say $P_{1}$ from above), one can in principle use Bayes theorem (2.1) to obtain the posterior $\tilde{P}(\theta \mid d)$ for a different prior $\tilde{p}(\theta)$ :

$$
\tilde{P}(\theta \mid d) \propto P(\theta \mid d) \frac{\tilde{p}(\theta)}{p(\theta)} .
$$

This prompts the following question: was it necessary to fully reanalyze the data to obtain the posteriors presented in Sec. 4? Couldn't one just re-weight previous posterior samples as in Eq. (5.1)? While this procedure is mathematically correct, it might be dangerous in practice, because the numerical algorithm used to sample $P$ might not cover the region of the parameter space that is of interest for $\tilde{P}$ densely enough. This issue was recently explored by Williamson et al. (2017): they find that re-weighted posterior samples might indeed lead to biassed conclusions. Re-weighting carries systematics which needs to be properly analyzed before Eq. (5.1) can be used in, e.g., hierarchical model selection schemes. Careful comparisons of our full reruns with re-weighted posteriors is a natural follow-up of the results presented by Vitale et al. (2017) and summarized here.

\section{Acknowledgements}

D.G. is supported by NASA through Einstein Postdoctoral Fellowship Grant No. PF6170152 by the Chandra X-ray Center, operated by the Smithsonian Astrophysical Observatory for NASA under Contract NAS8-03060.

\section{References}

Aasi, J., et al. 2015, CQG, 32, 074001

Abbott, B. P., et al. 2016a, PRX, 6, 041015

—. 2016b, PRL, 116, 241103

-. 2016c, PRL, 116, 061102 
-. 2017a, PRL, 118, 221101

-. 2017b, arXiv:1711.05578 [astro-ph.HE]

-. 2017c, PRL, 119, 141101

-. 2017d, PRL, 119, 161101

-. 2017e, ApJ, 848, L12

Acernese, F., et al. 2015, CQG, 32, 024001

Vallisneri, M., Kanner, J., Williams, R., Weinstein, A., \& Stephens, B. 2015, in Journal of Physics Conference Series, Vol. 610, Journal of Physics Conference Series, 012021

Vitale, S., Gerosa, D., Haster, C.-J., Chatziioannou, K., \& Zimmerman, A. 2017, PRL in press., arXiv:1707.04637 [gr-qc]

Williamson, A. R., Lange, J., O’Shaughnessy, R., et al. 2017, arXiv:1709.03095 [gr-qc] 